\title{
Lusioersily
}

\section{Literature Review on Transfer Learning for Human Activity Recognition Using Mobile and Wearable Devices with Environmental Technology}

\author{
Hernandez-Cruz, N., Lundstrom, J., Favela, J., McChesney, I., \& Arnrich, B. (2020). Literature Review on \\ Transfer Learning for Human Activity Recognition Using Mobile and Wearable Devices with Environmental \\ Technology. SN Computer Science, 1(66). https://doi.org/10.1007/s42979-020-0070-4
}

Link to publication record in Ulster University Research Portal

Published in:

SN Computer Science

Publication Status:

Published (in print/issue): 07/02/2020

DOI:

https://doi.org/10.1007/s42979-020-0070-4

\section{Document Version}

Author Accepted version

\section{General rights}

Copyright for the publications made accessible via Ulster University's Research Portal is retained by the author(s) and / or other copyright owners and it is a condition of accessing these publications that users recognise and abide by the legal requirements associated with these rights.

\section{Take down policy}

The Research Portal is Ulster University's institutional repository that provides access to Ulster's research outputs. Every effort has been made to ensure that content in the Research Portal does not infringe any person's rights, or applicable UK laws. If you discover content in the Research Portal that you believe breaches copyright or violates any law, please contact pure-support@ulster.ac.uk. 


\title{
Literature review on transfer learning for human activity recognition using mobile and wearable devices with environmental technology
}

\author{
Netzahualcoyotl \\ Hernandez* \\ Ulster University, Northern \\ Ireland, UK \\ hernandez_cruz-n@ulster.ac.uk \\ Jens Lundström \\ Raytelligence AB, Sweden \\ jensR.lundstrom@gmail.com \\ Ian McChesney \\ Bert Arnrich \\ Ulster University, Northern \\ Hasso Plattner Institut, \\ Ireland, UK \\ University of Potsdam, \\ ir.mcchesney@ulster.ac.uk \\ Germany \\ bert.arnrich@hpi.de
}

\author{
Jesus Favela \\ CICESE, Mexico \\ favela@cicese.mx
}

\section{ABSTRACT}

Activity recognition systems utilise data from sensors in mobile, environmental and wearable devices, ubiquitously available to individuals. It is a growing research area within intelligent systems that aims to model and identify human physical, cognitive and social actions, patterns and skills. They typically rely on supervised machinelearning approaches, in which the cost of gathering and labelling data is high due to the diverse, interleaved and dynamic nature of human behaviour. Transfer learning is an approach in which previously learned knowledge is utilised to model a new but related setting. For instance, it can reuse existing knowledge to recognise activities

${ }^{*}$ Corresponding author

Authors' addresses: Netzahualcoyotl Hernandez, Ulster University, Newtownabbey, Belfast, Northern Ireland, UK, hernandez cruz-n@ulster.ac.uk; Jens Lundström, Raytelligence AB, Klammerdammsgatan, Halmstad, Sweden, jensR.lundstrom@gmail. com; Jesus Favela, CICESE, Baja California, Ensenada, Mexico, favela@cicese.mx; Ian McChesney, Ulster University, Newtownabbey, Belfast, Northern Ireland, UK, ir.mcchesney@ulster.ac.uk; Bert Arnrich, Hasso Plattner Institut, University of Potsdam, Potsdam, Potsdam, Germany, bert.arnrich@hpi.de. 
2 - Netzahualcoyotl Hernandez, Jens Lundström, Jesus Favela, lan McChesney, and Bert Arnrich

performed by different types of users, using different sensor technologies and in different environmental conditions. As the adoption of Internet of Thing devices increases, mobile and wearable sensing is becoming pervasive, and more challenging behaviour recognition activities are being tackled. Yet, the availability of more data does not necessarily translates to better recognition models, if this data is not properly labelled. Thus, the importance of taking advantage of transfer learning to advance the field of activity recognition. This literature review summarises the transfer learning techniques and explores the benefits of combining mobile and wearable devices with environmental sensors in support of transfer learning. We also discuss the maturity of transfer learning by analysing the validation method used in the papers reviewed. Overall, 170 selected articles published between 2014 and 2019 were reviewed following the Okali and Schabram methodology. Findings show an increase of $41 \%$ of publications when comparing the output of 2019 against the average number of papers published in the previous five years (2014-2018). Inertial sensors such as accelerometers and gyroscopes, are the most frequently used. Feature and instance representation are mature techniques for transfer knowledge. Unsupervised learning across users is a typical application, and shallow techniques and active learning are areas of opportunity in transfer learning methodologies.

Additional Key Words and Phrases: transfer learning, human activity recognition, activities of daily living, mobile sensing, wearable computing, environmental technology

\section{INTRODUCTION}

Human activity recognition (HAR) is a research area that focuses on correctly classifying the activity that a human is performing given a set of data which captures human movement and environment interaction. HAR represents a key element in the growth of systems capable of providing personalised assistance [15] and health monitoring. Ordinarily, activity recognition uses supervised classification, that relies on self-report and limited in-person observations for data labelling, which is time-consuming and expensive in terms of human effort. In this regards, transfer learning seems like a promising approach, since it leverages unseen knowledge learned from previous user's profiles, sensing technology, and 
Literature review on transfer learning for human activity recognition using mobile and wearable devices with environmental technology $• 3$ task conditions [16].The use of transfer learning could thus help reduce the cost of training activity recognition models. Particularly as emerging wearable devices and Internet of Things (IoT) technologies increase the availability of data and the cost of labelling it in naturalistic conditions.

Knowledge to learn and to transfer can come from data collected using a plethora of available sensors such as mobile, environmental, and wearable sensors. Mobile [55] and wearable computing (e.g., smart-watches [8], and activity monitors (e.g., Fitbit) [54]) allow for collecting data on human activities anywhere and at any time, regardless of the environmental infrastructure [6]. Similarly, the static setting of smart environments helps to capture complex data that can enrich mobile and wearable contextual information [49]. Fixed sensing technology used in smart environments includes cameras [1], Radar signal [64], Wi-Fi antennas [22]. Such technologies make this approach more robust in terms of processing data and memory capacity compared to mobile and wearable devices. Approaches to HAR using mobile, wearable, and environmental (MWE) devices are varied [58, 62]. In healthcare [56, 59], for example, monitoring physiological performance while individuals conduct activities of daily living (ADL) opens the opportunity for short and long term monitoring, which facilitates health and wellbeing changes derived from medical treatment or rehabilitation interventions [36]. The maturity of these technologies and computational algorithms makes it possible to automatically learn and recognise activities from scenarios where training data is already available, enabling the possibility of taking advantage of the synergy of transfer knowledge across different technologies.

Transfer learning is an approach in which previously learned knowledge from one domain is utilised to understand another domain, e.g., activities collected from another user or environment by reusing existing knowledge [44]. In this context and according to Saeedi et al. [48], there are three essential questions to ask when developing a transfer learning solution. What to transfer?, How to transfer?, and When to transfer?. While previous literature reviews illustrate how transfer-learning benefits 
4 - Netzahualcoyotl Hernandez, Jens Lundström, Jesus Favela, lan McChesney, and Bert Arnrich activity recognition approaches in general terms [16], at the time of writing this paper, no one has systematically explored the synergy derived from combining mobile and wearable with environmental devices in terms of transfer learning. This study, therefore extends Saeedi's questions by introducing a fourth question (i.e., Where to transfer?) to explore the dimensions along which knowledge can be transferred such as user profile and technology.

When conducting this study, we observed that other literature reviews are constrained to some of the dimensions of transferring learning. For example, they may exclusively elaborate about the pose-base of the sensing devices worn [9], the subject's pattern behaviour [12], or specific technology domain like environmental sensing $[18,31]$. Other papers may discuss the trend classification techniques $[17,25]$.

Although many literature reviews are driven by conventional techniques to identify HAR [35] [32], none of them specialises in the transfer learning approach. Thus, given the relevance on the topic, here, we conduct an extensive literature search to explore how active in the research community working along with the different variants of transfer learning such as location, subject's profile, or technology. Rather than limiting our study to a particular HAR classification technique, we explore the interests of the community by offering quantitative results. Also, we examine the benefits and research maturity of transfer-based activity recognition of MWE sensing by bringing technological solutions to problems of HAR.

The literature analysis aims at addressing the following research questions:

- Is transfer learning being applied in HAR using data derived from MWE sensors?

- To what extent have transfer learning approaches explored the benefits of combining MWE computing?

- What are the essential technical features of transfer learning when applied to HAR - what, where, when and how? 
Literature review on transfer learning for human activity recognition using mobile and wearable devices with environmental technology $• 5$

- How mature is transfer learning in the context of HAR?

In the remainder of the paper, we examine the concept of transfer learning (Section 3) and describe the approach used (Section 4) to structure the literature review. Section 5 discusses the results whilst a discussion is drawn in Section 6. The paper concludes with the main findings in Section 7.

\section{TRANSFER LEARNING}

Transfer learning approaches can improve the robustness and scalability of activity recognition models by reducing the training effort to initiate new activity recognition by leveraging knowledge from previous tasks. Transfer learning assumes that a source and a target domain is present and that there exists some relationship between them, which enables meaningful knowledge transfer [16].

Pan and Yang [44] define transfer learning by formalising two components (i.e., Source/Target domain and task). Source domain can be denoted as $D_{S}=\left\{\left(x_{S_{1}}, y_{S_{1}}\right), \ldots,\left(x_{S_{n}}, y_{S_{n}}\right)\right\}$, where $x_{S_{i}} \in X_{S}$ is the data instance and $y_{S_{i}} \in Y_{S}$ is the corresponding class label. Similarly, target domain can be denoted as $D_{T}=\left\{\left(x_{T_{1}}, y_{T_{1}}\right), \ldots,\left(x_{T_{k}}, y_{T_{k}}\right)\right\}$, where the input $x_{T_{j}} \in X_{T}$ and $y_{T_{j}} \in Y_{T}$ is the corresponding output. Hence, given a specific domain, $D=\{X, P(X)\}$, a task consists of two components; a label space $Y$ and an objective predictive function $f($ ) (denoted by $T=Y, f()$ ), which is not observed but can be learned from the training data, which consists of pairs $x_{i}, y_{i}$, where $x_{i} \in X$ and $y_{i} \in Y$.

Transfer Learning, therefore, can be defined when given a source domain $D_{S}$ and a learning task $T_{S}$, a target domain $D_{T}$ and learning task $T_{T}$. Transfer learning aims to help improve the learning of the target prediction function $f_{T}()$ in $D_{T}$ using the knowledge in $D_{S}$ and $T_{S}$, where $D_{S} \neq D_{T}$, or $T_{S} \neq T_{T}$. For example, in the scenario in which we cannot guarantee access to labelled data in the target domain, transfer learning can leverage the labelled data from the source domains in order to map their knowledge to the target domain. 
In the context of HAR, transfer learning is a research area that addresses many different machine learning problems. Concept drift or change, which is a phenomenon in classification problems manifested through changes in either the initial distribution class or the unobserved samples [24]. Activity discovery, which is a technique capable of recognising an unbounded pre-defined set of activities [16], using for example unsupervised clustering [34] (feature-based approaches), motif discovery techniques [7, 39], or topic models [29,50]. Active/online/incremental learning and learn-on-the-go are strategies that extend their knowledge based on the acquisition of new activity recognition insight upon conditioned iterations executed only when new knowledge can improve the performance of the current model. Such an approach generates activity recognition models with small training datasets, and decides whether to query an update of the model upon the arrival of each new sample received in real-time [45]. One example is the Adaptive Resonance Theory (ART), which is a cognitive and neutral theory solving the problem of how a machine can learn knowledge quickly from the new data without forgetting previously learned knowledge. Related is the Self-supervised Predictive ART, which is a neural network architecture for supervised incremental learning problems, that partially learns from labelled inputs, and enriches itself according to unlabelled inputs containing additional novel features [13].

\section{METHODOLOGY}

This study was conducted as a systematic literature review following Okali and Schabram's step-by-step guide which is an approach intended to assure rigorous review to produce a comprehensive summary and discussion of the existing literature in the particular area of information systems (IS) [42]. Following this approach, we have conducted our study based on eight steps: Purpose of the literature review, Protocol \& training, Searching the literature, Practical screening, Quality appraisal, Data extraction, Analysis of findings, and Writing review. 
Literature review on transfer learning for human activity recognition using mobile and wearable devices with environmental technology $• 7$

\subsection{Eligibility Criteria}

The interest literature review focuses on original papers investigating MWE computing, transfer learning, and activity recognition. Surveys and reviews papers were excluded to avoid duplication as well as studies consisting of activity recognition techniques overlooking transfer learning approaches, and papers lacking scientific format. The publications were restricted to journals and conference proceedings written in the English language.

\subsection{Information Sources and Search Strategy}

The published works were identified by conducting a systematic literature search in IEEE Xplore, Scopus, ACM, and Web of Science. We used these resources due to their technological and scientific orientation.

The literature search consisted of three key concepts, i) transfer learning, ii) activity recognition, and iii) technology (i.e., mobile, wearable, and environmental computing). The literature search was conducted using the following keywords:

(i) "transfer learning", "active learning", "learning algorithms" "learning to learn", "information transfer", "life-long learning", "knowledge transfer", "knowledge discovery", "inductive transfer", "transfer function", "context-sensitive learning", "meta-learning", "multiple-transfer", "self-taught learning", "multitask learning", "domain adaptation", "learning systems", "covariate shift", "multi-source", "transfer functions", "supervised learning", "unsupervised learning", "data driven", "knowledge driven".

(ii) "activity recognition", "multilabel recognition", "action recognition", "assisted living", "lifelogging", "human behaviour monitoring", "home automation", "context-awareness", "behaviour analysis", "activity prompting", "ambient assisted living". 
8 - Netzahualcoyotl Hernandez, Jens Lundström, Jesus Favela, Ian McChesney, and Bert Arnrich

(iii) "wearable", "smartphone", "smart phone", "mobile", "smartwatch", "smart watch", "cellphone", "gadget", "monitoring", "mHealth", "pervasive computing", "mobile computing".

As technology becomes more accessible as time passes, it has been observed how in recent years device sales has increased, from 265 to 347 million units in 2016 and 2017. The forecast is for 504 million devices to be commercialised by $2021^{1}$. Market sales report an increase from 1.48 to 5.8 millions of U.S. dollars since 2014 to $2018^{2}$. In this regards and due to the introduction of sensor-equipped smartphones in 2007 (i.e. the first iPhone), the query was restricted to a period from 2007 (inclusive) to 2019 (inclusive). The last literature search update was conducted in December 2019.

\subsection{Paper Selection}

As presented in the flow diagram of the systematic review depicted in Figure 1. The selection criteria consisted of articles that describe the use of transfer learning and sensing technology in the context of MWE computing for HAR.

There were 2,155 papers retrieved out of the four referenced databases. We excluded 425 papers due to duplication across the databases. The titles of all papers were manually assessed, aimed at identifying irrelevant papers; 495 papers were excluded in this way. The abstract of relevant papers $(n=727)$ was screened for eligibility and labelled as relevant, irrelevant, unclear. To depict the most recent contribution from the research community, this review focuses on papers published from 2014 to 2019 inclusive $(n=562)$. The selected papers were read in full to assure that the eligibility criteria were satisfied. Through this process, only 170 articles were considered as relevant, hence included in this particular review.

\footnotetext{
${ }^{1}$ Accessed July 31st, 2019: www.techcrunch.com/2017/08/24/global-wearables-market-to-grow-17-in-2017-310m-devices-sold30-5bn-revenue-gartner

${ }^{2}$ Accessed July 31st, 2019: www.statista.com/statistics/302482/wearable-device-market-value/
} 
Literature review on transfer learning for human activity recognition using mobile and wearable devices with environmental technology • 9

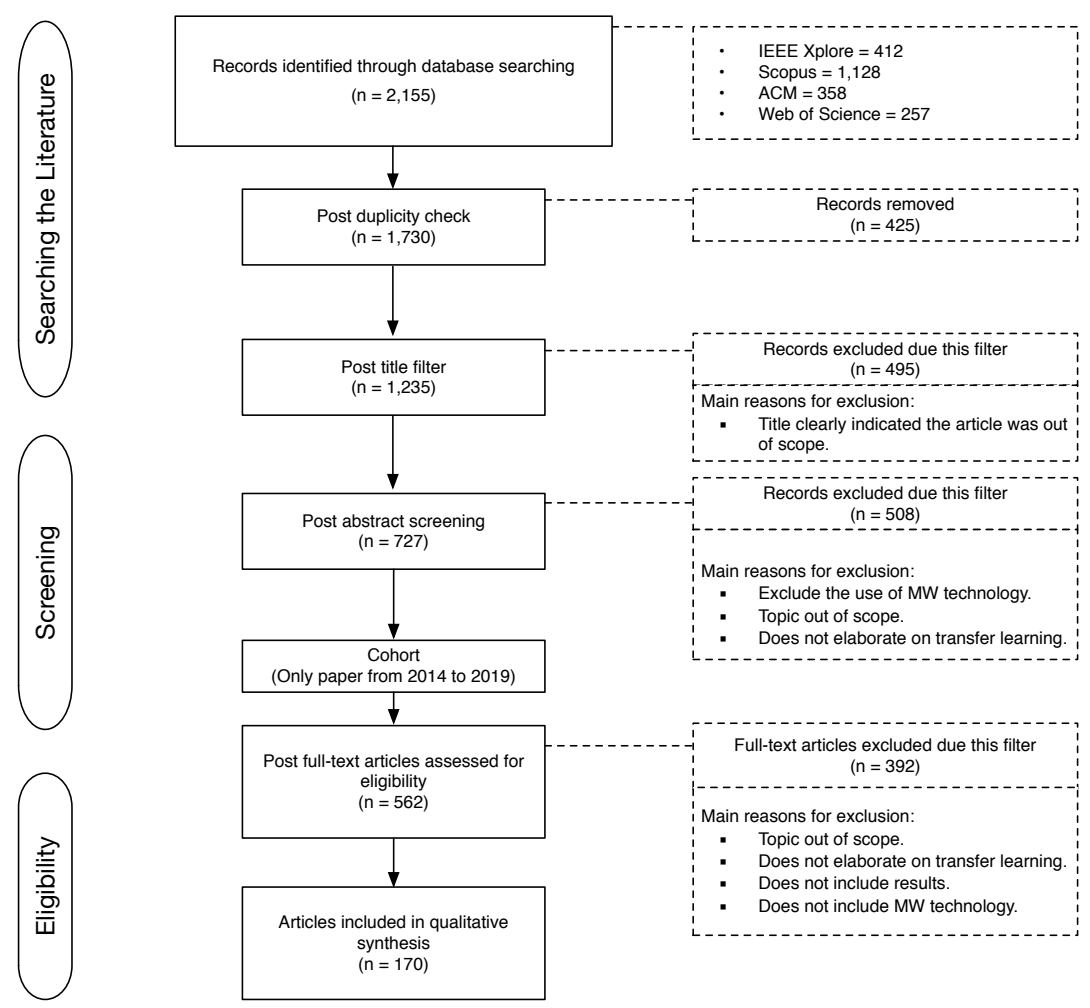

Fig. 1. Flow diagram of the literature search based on the Okali and Schabram methodology.

The included studies were reviewed in detail to glean relevant information such as technology used for sensing (smartphone, wearable, environmental), type of sensing condition (naturalistic/scripted/controlled), type of sensor, location of the sensor, and transfer learning approach. We have classified the research studies based on their technology trend by creating three categories. The Mobile Devices category represents studies in which Smartphones are the key elements of a study, the Wearable Devices category represents studies utilising any technology meant to be worn, such as smart-watches or Fitbits. The Environmental Technology category represents objects enabled with embedded sensing technology and environmental technology to monitor indoor activities (e.g., smart homes). Note that given the research interest that drives this review, the environmental technology category exclusively considered 
10 - Netzahualcoyotl Hernandez, Jens Lundström, Jesus Favela, Ian McChesney, and Bert Arnrich environmental approaches which integrate mobile or wearable technology. Any disagreements in category allocation were resolved through a discussion among the authors.

\section{RESULTS}

In this section, the findings of the research questions are described in four sub-sections. The first sub-section introduces general results to illustrate the adoption of transfer learning approaches in the scope of MWE technology. We then, present the results on how transfer learning can be utilised to derive HAR models using data from MWE technology. In the context of transfer learning, the third sub-section explores the synergy of combining MWE technologies for HAR. The last section presents the impact that transfer learning has had in recent years in HAR as well as describing the maturity of transfer learning techniques based on the data gathering methodology followed in the study. Due to the fact that papers describe multiple experiments, our statistical results quantify the analyses of the experiments rather than the number of papers. Hence, one paper can be represented two or more times (i.e., one per experiment conducted).

\subsection{Is transfer learning being applied in HAR using data derived from MWE sensors?}

On average there are 28 papers published and 44 experiments conducted per year from 2014 to 2019. As depicted in Figure 2, there were approximately 23 papers published per year in the first four years, and almost twice as much work carried out in 2019. In the last two years there is a substantial increase in interest in the field with 92 (54\%) papers and 148 experiments published. 
Literature review on transfer learning for human activity recognition using mobile and wearable devices with environmental technology $\bullet 11$

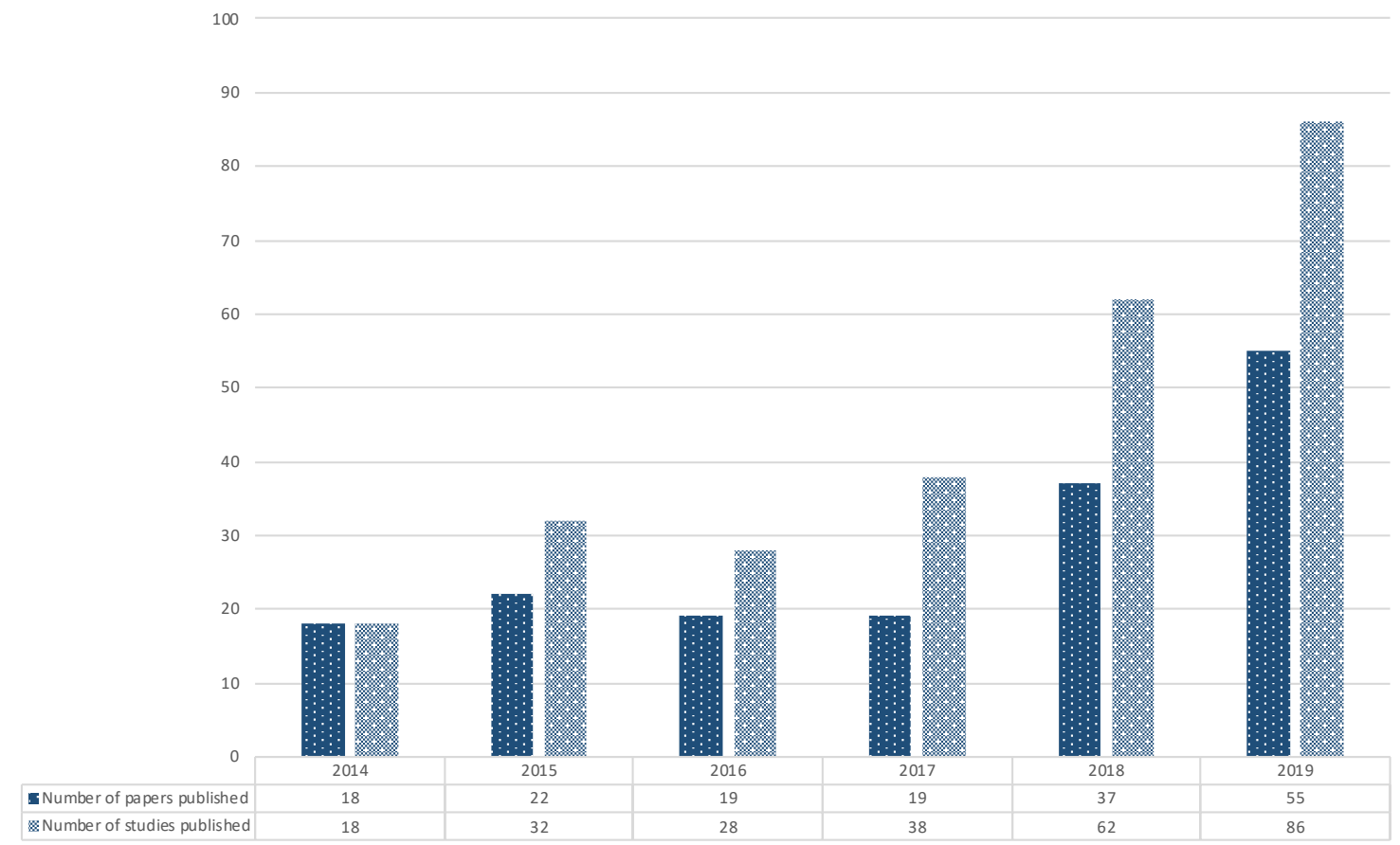

Fig. 2. Relationship between the number of research studies reviewed compared against the number of paper published from 2014 to 2019.

5.2 To what extent have transfer learning approaches explored the benefits of combining MWE computing?

Overall, there is a total of 170 papers published from 2014 to 2019 , which introduce 264 studies using data gathered with MWE computing technology. As depicted in Figure 3, data from the Wearable Devices category is most frequently used with a total of 162 studies (57\%), followed by data from the Mobile Devices and Environmental Technology categories with a total of 95 (34\%) and 26 studies (9\%), respectively.

The most frequently used sensor modalities in transfer learning studies in HAR are accelerometers and gyroscopes. As shown in Figure 4, 208 studies (53\% of the studies) utilised off-the-shelf sensors such 
12 - Netzahualcoyotl Hernandez, Jens Lundström, Jesus Favela, Ian McChesney, and Bert Arnrich

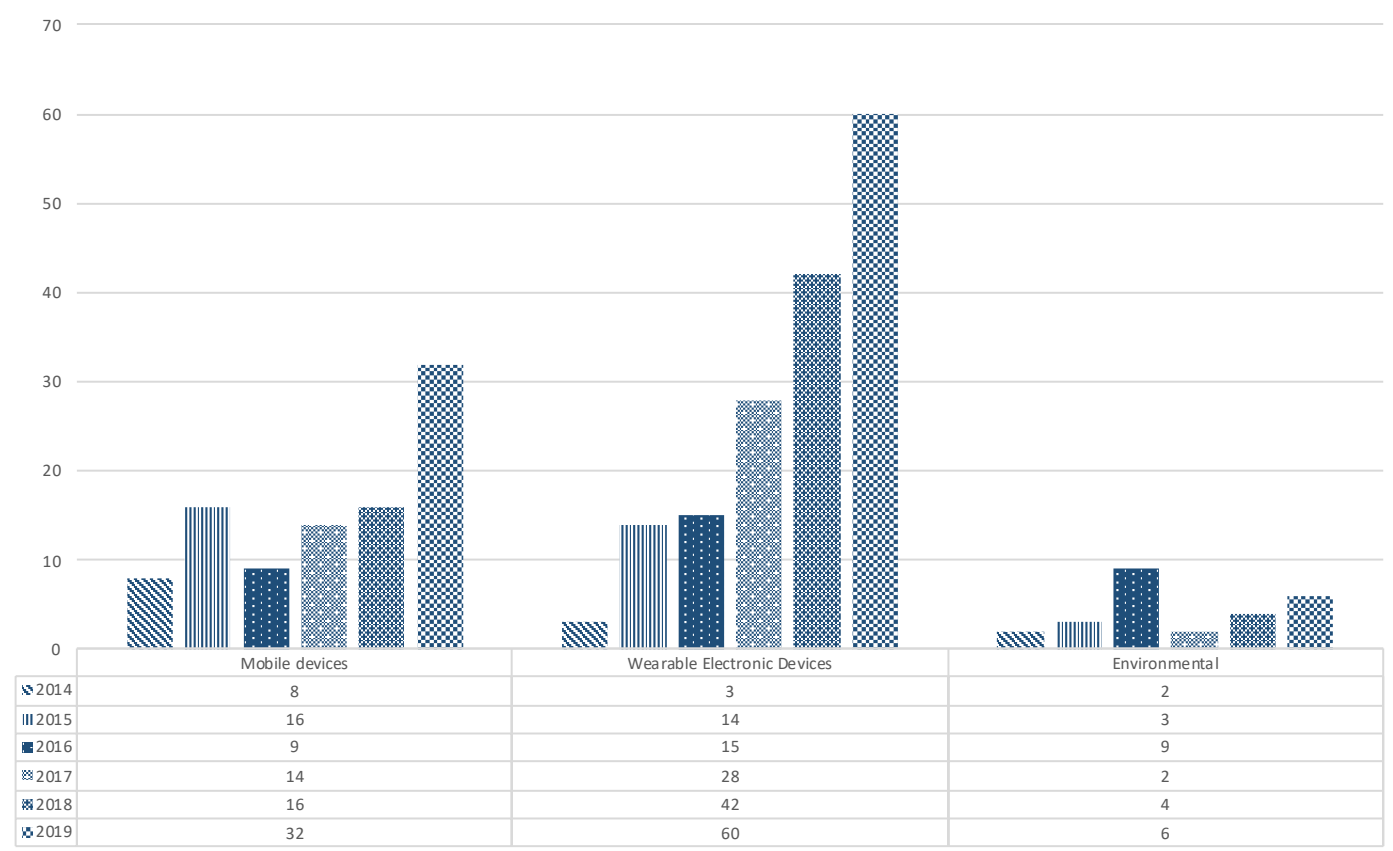

Fig. 3. Comparison of the number of studies conducted within each type of device (MWE) used to gather sensor data in the papers published from 2014 to 2019.

as accelerometers, followed by the use of gyroscope with 95 studies (24\%). Two of the less explored sensors are thermometers and barometers with a single study adopting each technology.

In Figure 5 the technological tendency of the studies mentioned above is illustrated. Microcomputers such as Arduino sensor, sensing module boards, and Shimmer are the most popular selection of devices in HAR research, up to $51 \%(\mathrm{n}=156)$ of the studies have adopted them. These are followed by Smartphones $(29 \% ; \mathrm{n}=89)$, and Smartwatches $(8 \% ; \mathrm{n}=25)$. The result also shows that acceleration is the feature which is examined the most (208 studies), followed by gyroscope $(\mathrm{n}=95)$, and magnetometer $(\mathrm{n}=39)$ sensing technology. 
Literature review on transfer learning for human activity recognition using mobile and wearable devices with environmental technology $• 13$

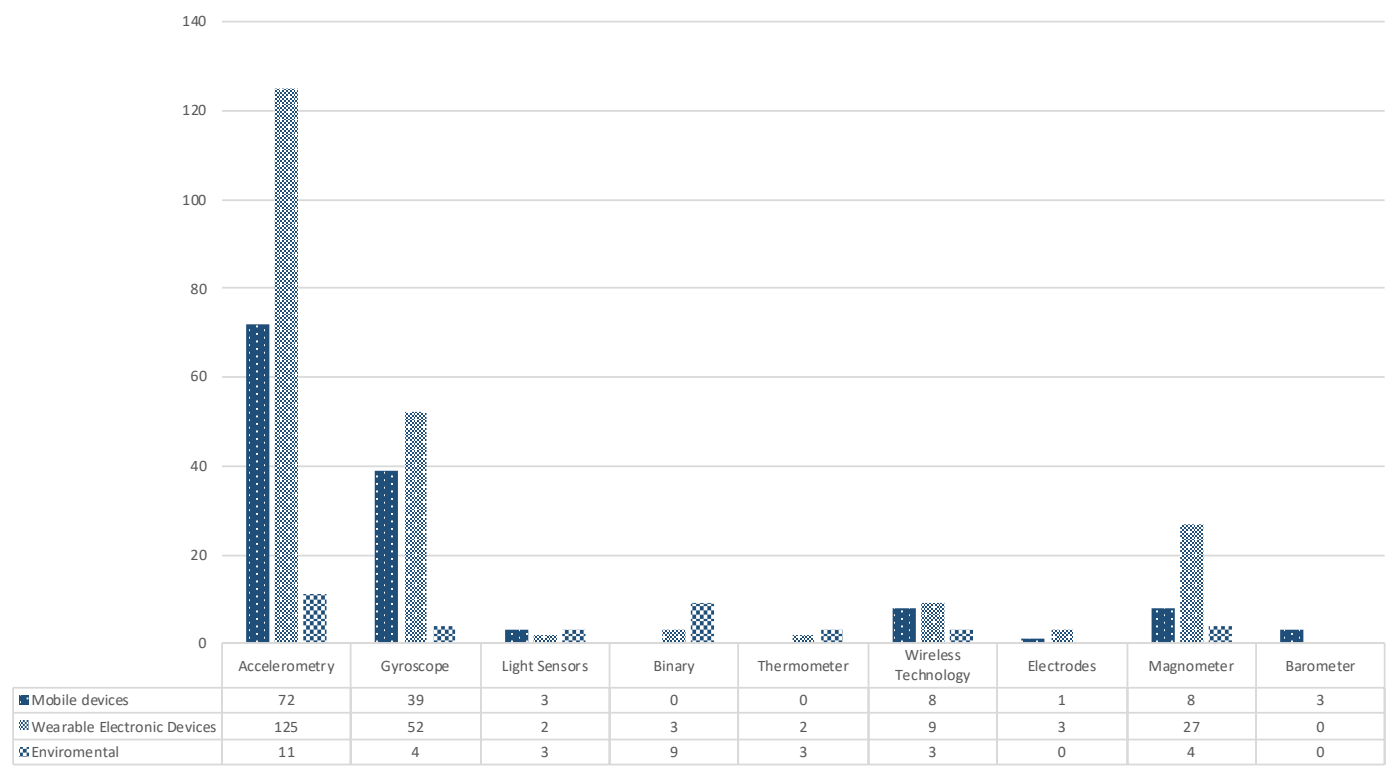

Fig. 4. Sensor technology utilised in the reviewed studies grouped by sensing technology and MWE computing approach.

\subsection{Which are the essential technical features of transfer learning when applied to HAR - what, where, when and how?}

In this section, we present results from four essential aspects when developing a transfer learning solution for HAR. As illustrated in Figure 6, the first question (i.e., What to transfer?) refers to the transfer elements being transferred, for instance, feature, instance, and parameter representation. This is followed by the dimensions through which data transfers (i.e., Where to transfer?) such as user or sensing technology. The next aspect addresses the temporal criteria by which labelled data transfer (i.e., When to transfer?); this includes inductive and transductive learning, as well as Teacher/Learner. The final aspect (i.e., How to transfer?), relates to the technical aspects required to solve transfer learning problems from the classification perspective, for instance by utilising techniques such as deep learning, concept drift, or shallow methods. The following sub-sections provide further details. 
14 - Netzahualcoyotl Hernandez, Jens Lundström, Jesus Favela, Ian McChesney, and Bert Arnrich

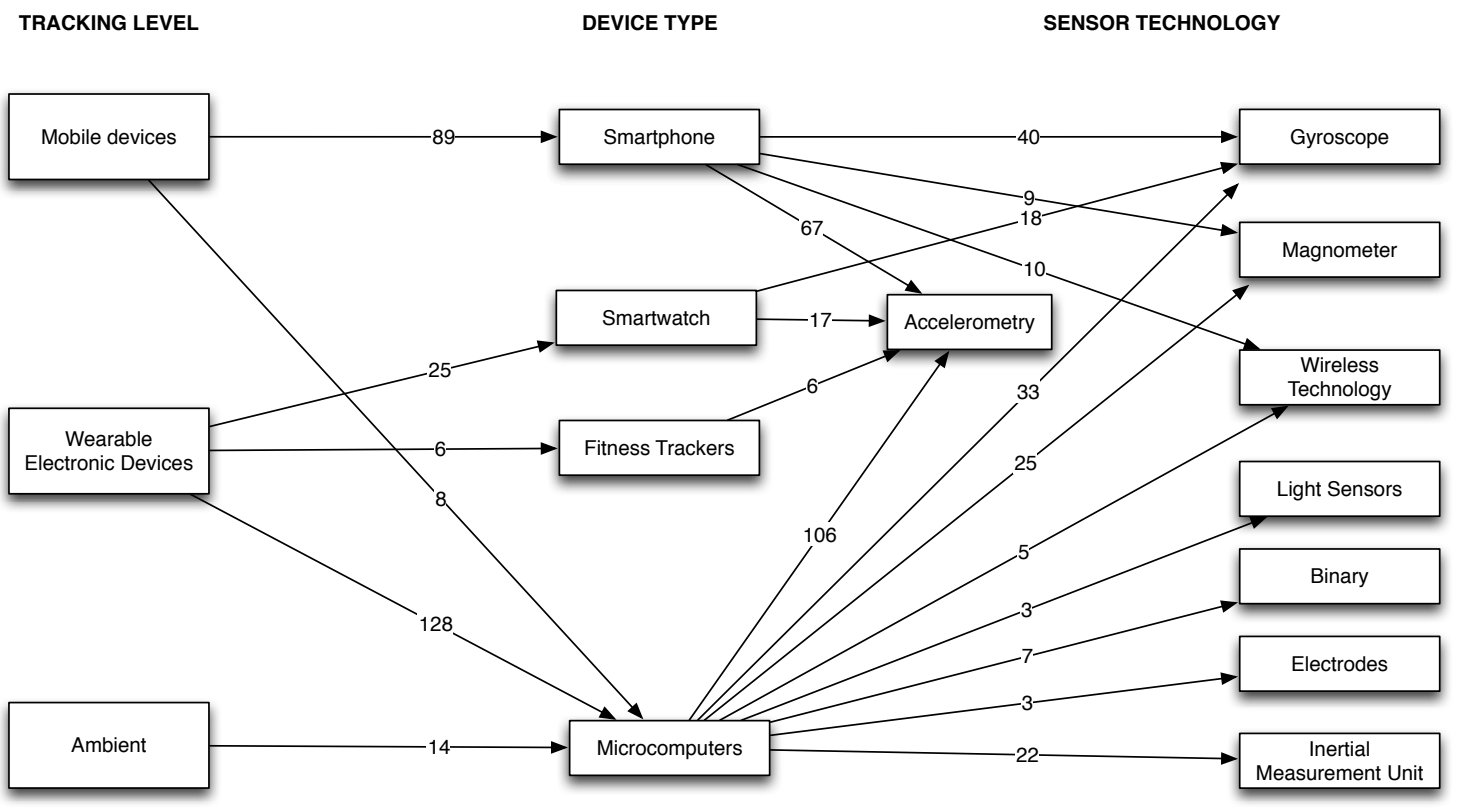

Fig. 5. Relationships of the sensor technology adopted to build the HAR model. Each unit represents one study in which the sensing technology has been utilised. Granularity is presented from the general (left) to the specific (right).

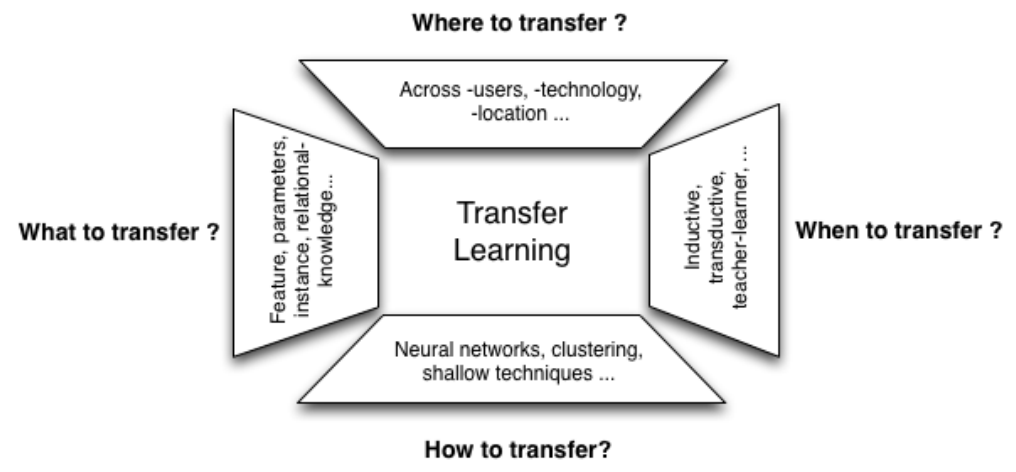

Fig. 6. Four questions used to characterise transfer learning approaches.

5.3.1 What to transfer? We classify the studies into four different transfer learning approaches [16].

Feature-representation transfer, which reduces the differences between the source and target feature spaces by mapping the source feature space to the target feature space [60]. Instance transfer that 
Literature review on transfer learning for human activity recognition using mobile and wearable devices with environmental technology $\bullet 15$

reuses the source data to train the target classifier, usually by re-weighting the source instances based on a given metric [26]. Parameter transfer, which learns parameters of trained models which are shared between the source and target tasks [11]. Relational-knowledge transfer, which applies to problems in which the data is not independent and identically distributed as is traditionally assumed but can be represented through multiple relationships [43].

In Figure 7, it can be observed that instance transfer and feature-representation transfer are the two most common techniques utilised in HAR research studies. Instance transfer is used in $54 \%$ of the studies $(n=143)$, and feature-representation transfer $31 \%(n=82)$. The results show that parameter transfer $(n=28)$ and relational knowledge transfer $(n=10)$ techniques are areas of opportunity with fewer studies conducted in recent years.

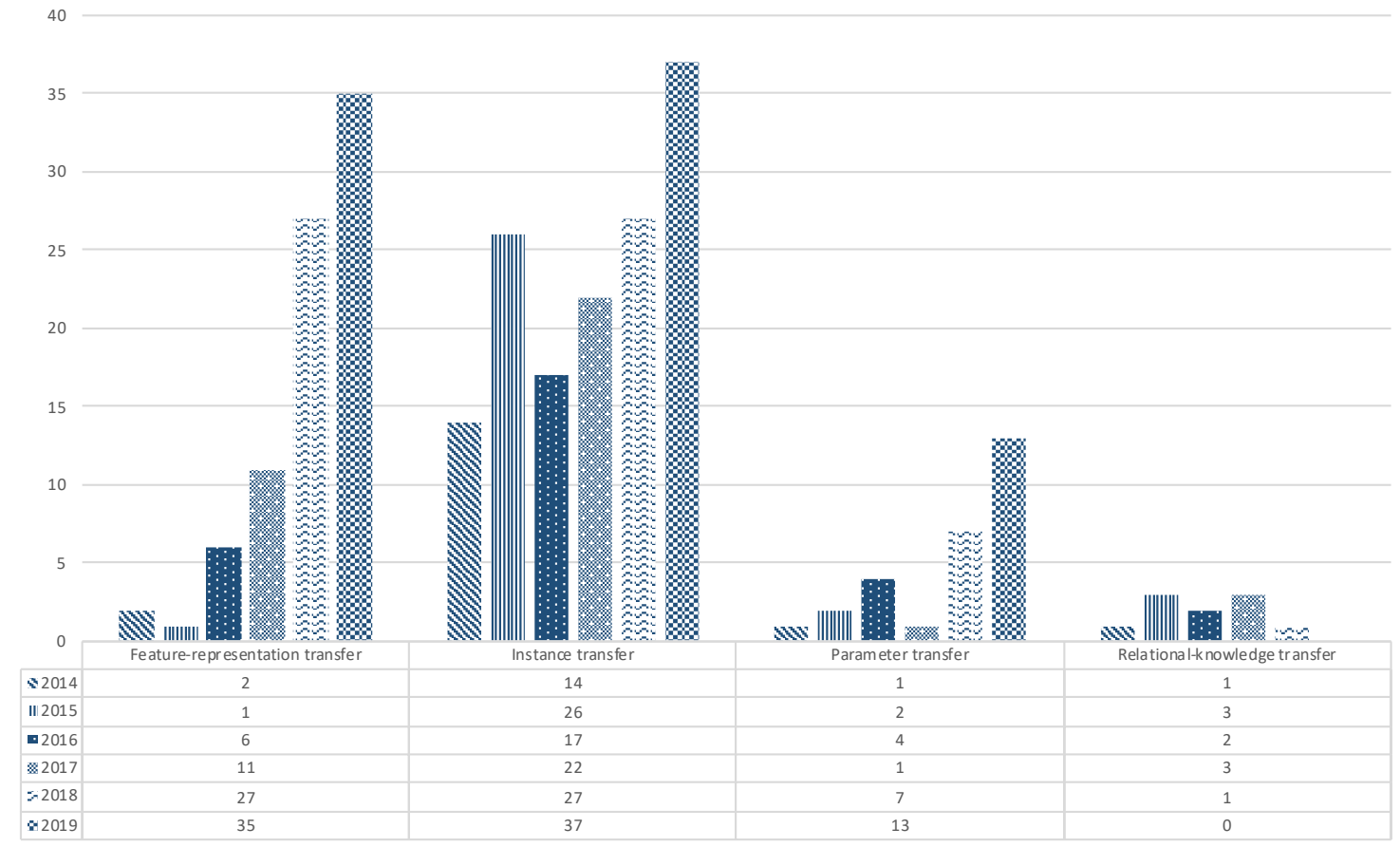

Fig. 7. Comparison between the number of studies adopting one of the different transfer level techniques grouped by year. 
5.3.2 Where to transfer? To answer this question, the studies were grouped based on the transfer learning interacting characteristics of the source and target entities. We identified 6 types of transfer learning according to these criteria: Across task (AT), that aims at labelling the data from one task by utilising the data from another task where $T_{S} \neq T_{T}$; Across location (AL), which refers to labelling unseen activities in data by making use of the same worn sensing technology located in both source and target domains (i.e., $D_{S}=D_{T}$ ) where $T_{S}=T_{T}$; Across sensor (AS), which focuses on labelling data utilising the data from different technology where $T_{S}=T_{T}$; Across environment (AE), that represents the capacity of labelling data from one domain context to another, in which the activities (i.e., $T_{S}=T_{T}$ ) and technology are similar, where $D_{S} \neq D_{T}$; Across sampling rate (AR), that aims at labelling data utilising data from same technology but different technical characteristics such as sampling rate, where $D_{S}=D_{T}$ and $T_{S}=T_{T}$; and Across user (AU), which represents the capacity to generalise label data from a different user where $D_{S}=D_{T}$ and $T_{S}=T_{T}$.

As depicted in Figure 8, the AU and AL transfer learning are the most common transfer dimensions, which are utilised in 190 and 40 studies respectively (representing 70\% and 15\% of the studies analysed in this review). In contrast, $\mathrm{AR}$ and $\mathrm{AE}$ are dimensions that have been poorly explored with only 10 (4\%) and 4 studies (2\%), respectively.

W.3.3 When to transfer? To answer this question, we grouped the studies into four categories: Inductive learning (IL), which implies that some labelled data is available in both the target and source domains [40]; Teacher/Learner (T/L), in which no training data is directly available. Instead, a previously-trained classifier (the Teacher) is introduced which operates simultaneously with the new classifier to be trained (the Learner) and provides labels for observed data instances [27]. Transductive learning (TL), which implies that labelled data is available only in the source domain [47]; and Unsupervised learning (UL), which implies that no labelled data is available for either the source or target domains [23]. 

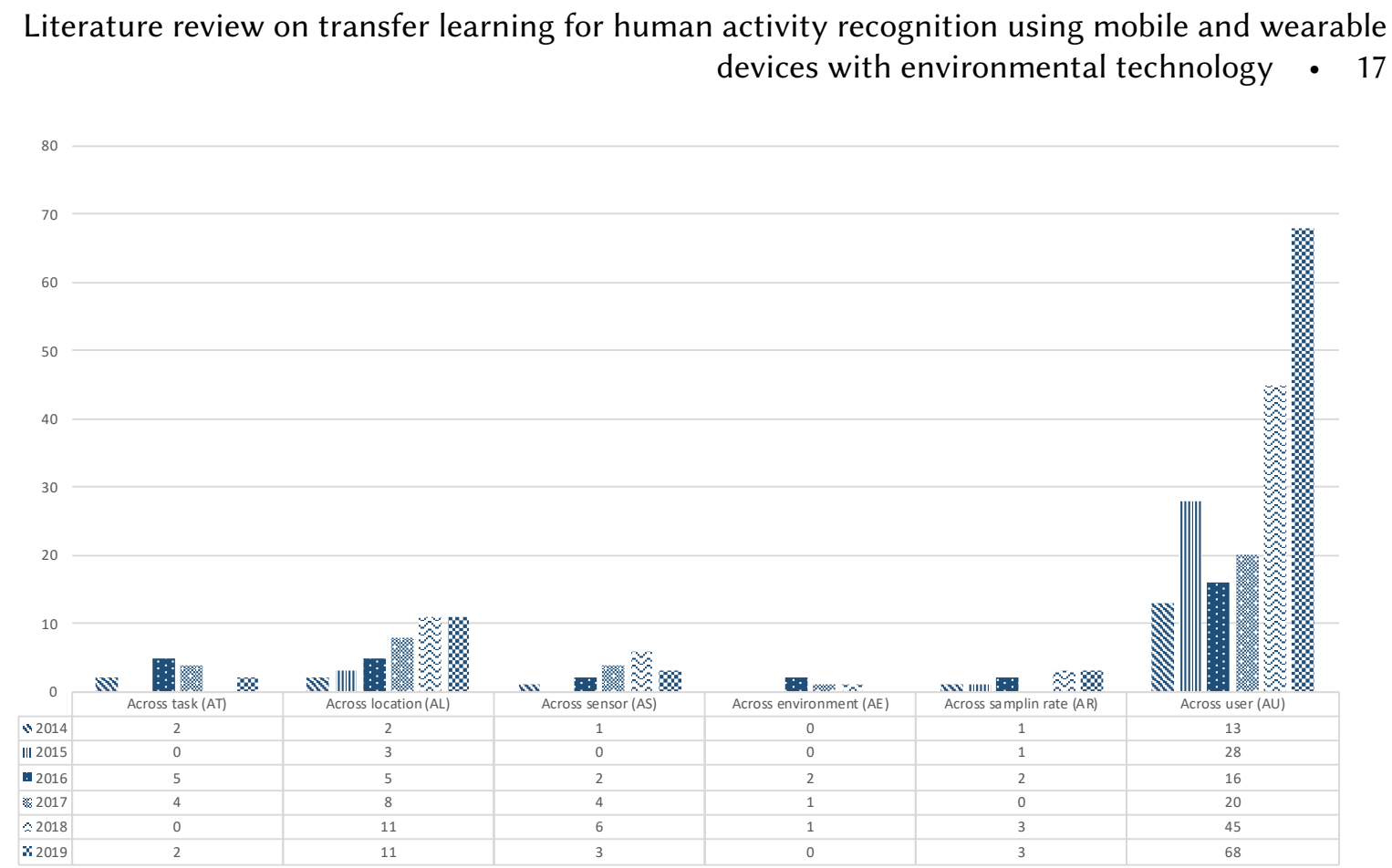

Fig. 8. Number of studies grouped by their transfer dimension, which is characterising conditions of the source and target domains.

In Figure 9 we observe that UL has the highest quantity with 149 studies over the six years (corresponding to $56 \%$ of the studies reviewed). In contrast, $\mathrm{T} / \mathrm{L}$ is an approach recently adopted by the community, with only four studies conducted so far. The number of studies using TL and IL techniques rates overall the same by covering approximately the $21 \%$ of the studies reviewed.

5.3.4 How to transfer? To answer this question, we classified the studies focused on their classification approach such as Deep Convolutional Networks (CNN) [41] and Deep Neural Network (DNN) [38], Clustering [30], Graph-based algorithms [52], Active learning [53], Multi-view learning [57], and Ontological reasoning [14]. We also considered experimental procedures based on shallow techniques; which is a term used to contrast with alternate deep learning architectures [63].

As depicted in Figure 10, Shallow classifiers rate as the highest technique explored for HAR with 100 studies (36\%). Similarly, Active learning and Deep Neural Networks are areas commonly explored 


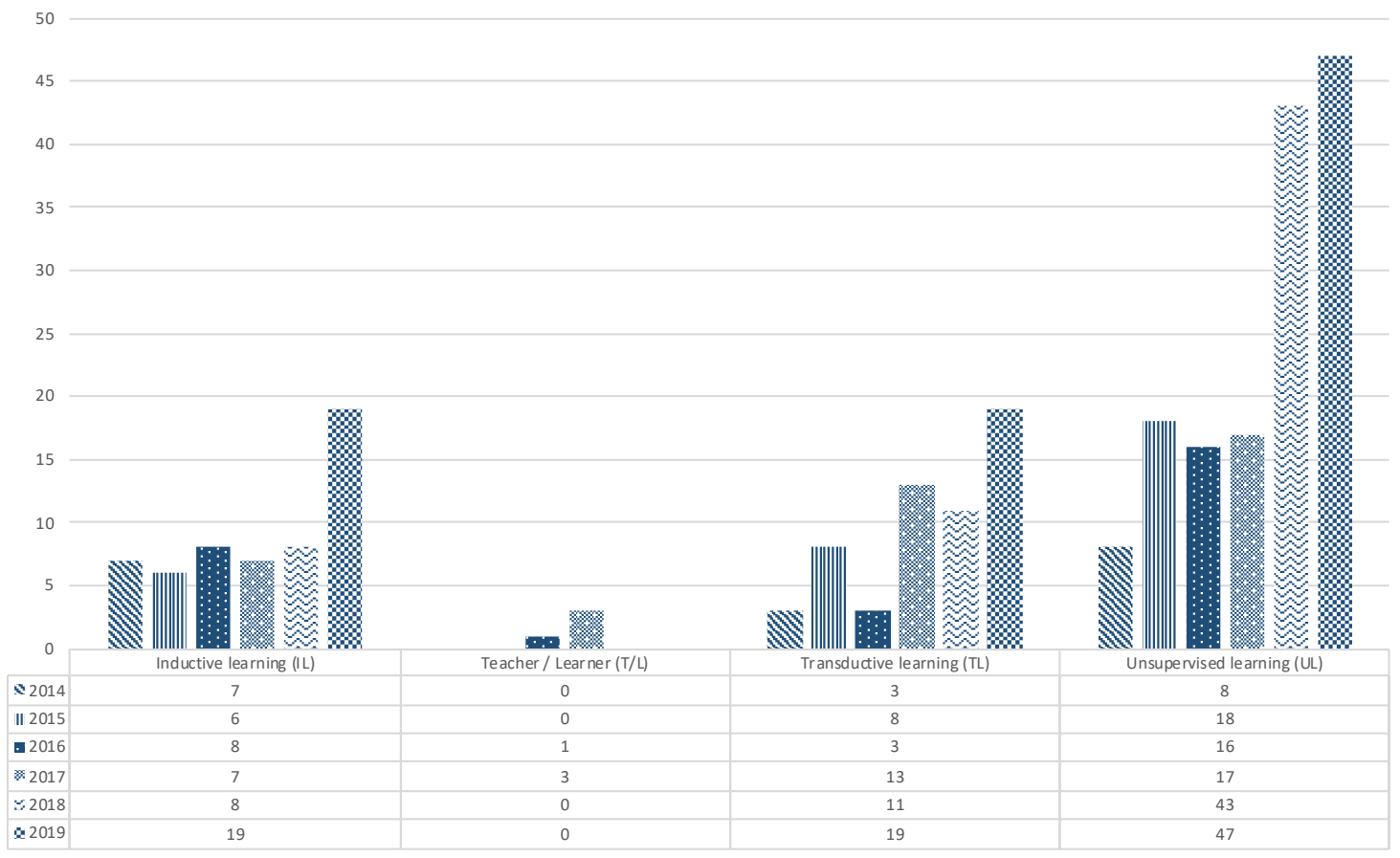

Fig. 9. Number of studies characterised by how labelled data is acquired to train the HAR models.

with about $21 \%(\mathrm{n}=58)$ and $16 \%(\mathrm{n}=44)$ studies, respectively. In contrast, the Concept $\mathrm{drift} / \mathrm{shift}$ and Semantic/Ontological reasoning techniques are novel approaches explored in three and seven of the studies from this review.

\subsection{How mature is transfer learning in the context of HAR?}

To answer this question, we investigate two topics. Conditions (e.g. controlled or naturalistic) in which data was collected to build and test each transfer learning approaches for HAR, and the contribution each study reported in their paper. Firstly, we classify the studies based on the characteristics and constraints of how the subjects undertake their activities at the data collection stage. There are four categories documented in the literature, i) Naturalistic conditions, where the subjects perform their ADL in either their homes or at smart living spaces as they usually conduct their day-to-day activities 
Literature review on transfer learning for human activity recognition using mobile and wearable devices with environmental technology $• 19$

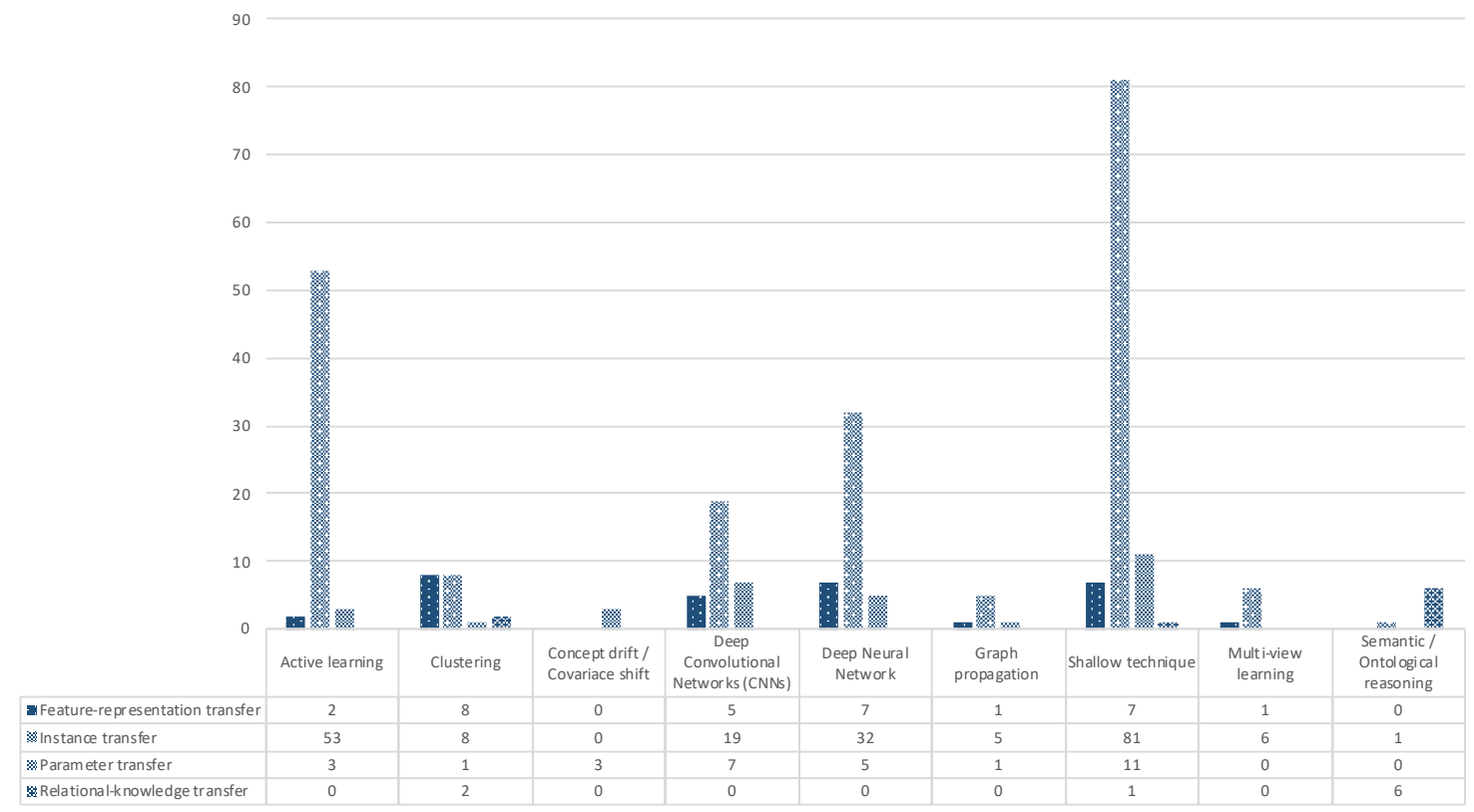

Fig. 10. Number of studies grouped by classification techniques and transfer learning approach.

[37]. ii) Naturalistic environment with controlled activities, where subjects perform their ADL as if they were interacting in their day-to-day environment, by following a written script indicating the activities to be performed, in either their homes or smart living spaces [10], iii) Controlled conditions, in which the subjects develop their ADL in a laboratory (e.g., smart living space) and follows a scripted set of activities [51], and iv) Synthetic, which represent data automatically generated by a computational program settled up a-priori with a set of parameters assigned to a particular activity [33,61].

In Figure 11, we observe that Controlled conditions studies are the most popular approach in research studies covering an overall of $45 \%$ of the studies (117 studies), followed by studies conducted in naturalistic environments with scripted activities having 108 studies (41\%). A total of 36 studies were identified as being conducted under naturalistic conditions, which represent $14 \%$ of the studies. Only a single study was conducted using synthetic data. 


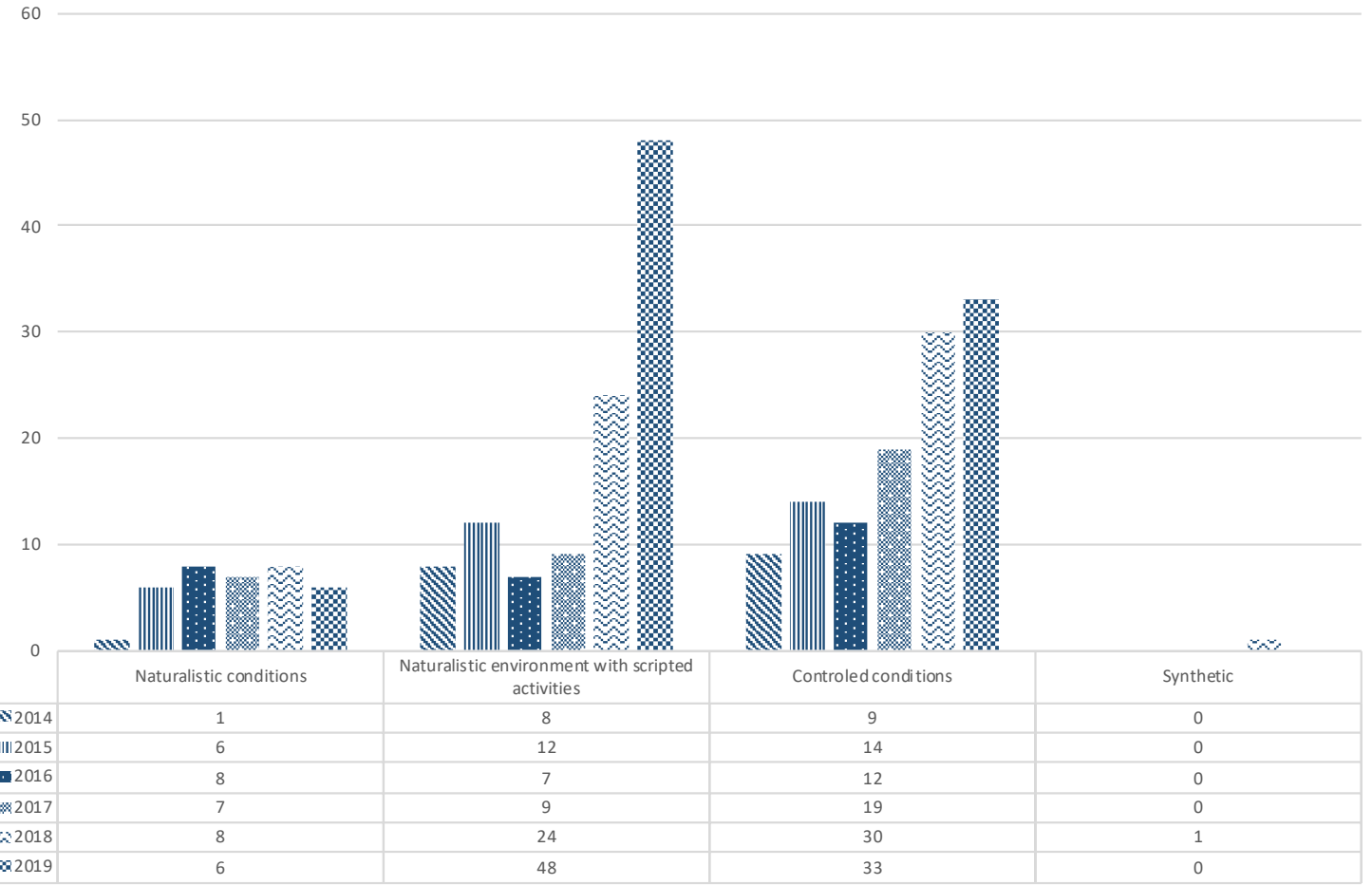

Fig. 11. Number of studies grouped by the data collection characteristics in which subjects developed their ADL.

Secondly, we classified the type of contribution a paper belongs to according to five groups. Algorithms, where the contribution consists of a sequence of algebraic formulas and/or logical steps to calculate or determine a given task. Methodology, which describes a procedure consisting of a sequence of algorithms and logical steps to calculate or determine a given task. Data Collection, which consists of rich/relevant data of daily living collected in the study. A framework, which introduces general-purpose computational planning and architecture to cope with the challenges of recognising ADL. Systems Integration, which describes mechanisms for combining separately developed modules, components, or subsystems so that they work together as a complete system.

Figure 12 depicts 254 papers that build upon methodologies to address transfer learning tasks, which corresponds to $58 \%$ of the total contribution reported, followed by frameworks (106 studies; $24 \%$ ) and 
Literature review on transfer learning for human activity recognition using mobile and wearable devices with environmental technology $\quad 21$

algorithm proposals (61 studies; $14 \%$ ). In contrast, only three studies that emerged from this systematic literature review have introduced new data sets, and four studies have developed systems as final solutions for HAR.

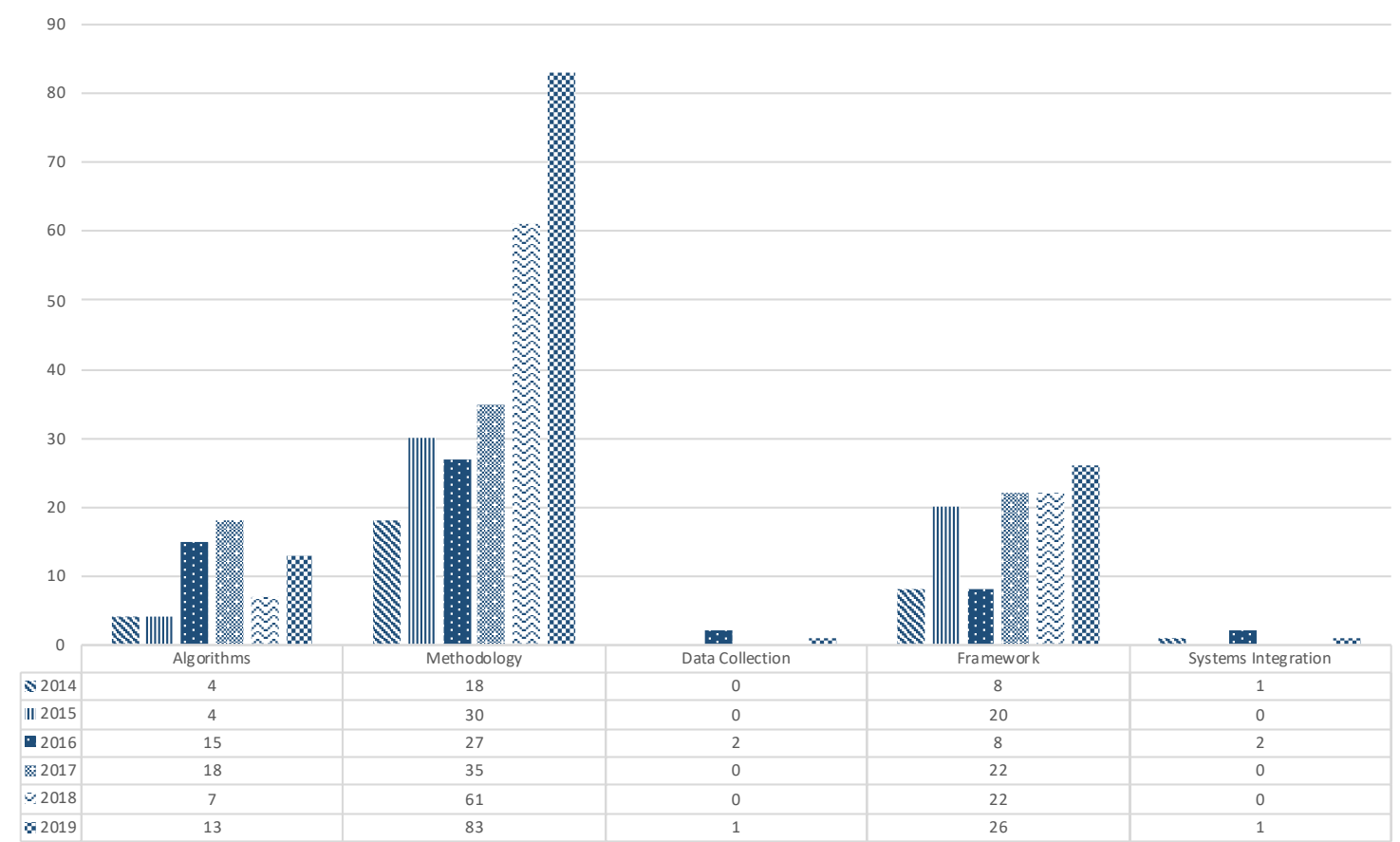

Fig. 12. Comparison among the number of studies grouped by their contribution towards HAR.

\section{DISCUSSION}

Given that MWE technology is already ubiquitous and is available to be either carried, worn, or embedded within daily-life objects, it is not surprising to conclude a growth in the use of data from these devices in HAR research. We found an increase of approximately $41 \%$ of publications when comparing the output of 2019 against the average number of papers published in the previous five years (i.e., 2014-2018). These results concur with the opinion of Appelboom et al. [3], who state that the feasibility of wearable technology relies on its practicability to be worn, increasing its engagement to 
22 - Netzahualcoyotl Hernandez, Jens Lundström, Jesus Favela, lan McChesney, and Bert Arnrich

the user. In this regard, we expect research in HAR using data from wearable devices to rise as their use becomes pervasive. Even more, as new wearable devices become available, with new sensing and connectivity capabilities, the use of transfer learning to quickly adapt to this new type of data would require additional research in transfer learning for HAR.

Sensing technology used in smart environments includes cameras [1], RFID tags [22], Wi-Fi antennas [22], among other technologies that have more robust processing and storage capacity compared to mobile and wearable computing. This enables systems to respond to natural interactions proactively. Environment-based systems are constrained to a static setting but help to capture complex data that can enrich contextual information [49]. In contrast, advances in mobile and wearable computing as well as the technology embedded in smartphones [55], smart-watches [8], and activity monitors (e.g., Fitbit) [54] allow for the recognition of human activities anywhere and at any time, regardless of environmental infrastructure [6]. These characteristics facilitate the user with the adoption of technology, offering many opportunities for the private and public sector to invest. As indicated in Section 5.2, mobile and wearable approaches are more frequently being used in HAR research than environmental technology over the last six years (i.e., 2014-2019).

Our results also suggest that accelerometer and gyroscope are the most common sensor modalities used in transfer learning research for HAR. In the context of pervasive and ubiquitous computing, inertial sensors are dominant in inferring motor activities such as walking, standing, sitting. Their pervasive presence in mobile and wearable computing make them practical in terms of mobility, diversity of sensor technology, and user-friendly interfaces which benefit the deployment of new devices and services.

The answer to one of our four research questions, "What to transfer?" showed that feature representation and instance transfer techniques represent a popular approach to data exploration. On the 
Literature review on transfer learning for human activity recognition using mobile and wearable devices with environmental technology $• 23$

other hand, there have been few studies on parameter and relational-knowledge transfer. In particular, the recent increase in the number of studies on parameter transfer could indicate that this is an expanding area of investigation and may contain opportunities for new research. With regards to "How to transfer?" the results suggest that the most popular options have been traditional methodologies such as shallow learning and active learning; while the approaches that have recently grown include techniques driven towards semantic/ontological reasoning, clustering, and covariant shift. The results on "When to transfer?" show that the most explored option relies on unsupervised learning, whereas Teacher/Learner techniques represent a novel approach in transfer learning. Finally, on the issue of "Where to transfer?", across user is the transfer dimension most frequently used, whereas across task, across the environment, and sampling rate are transfer learning dimensions poorly explored, indicating promising opportunities for the research community in terms of novelty.

We have observed that the research community is actively working in shallow methodology, while some of the advantages drive toward the small amount of training data and computation power consumption, drawbacks lie upon the constraint of pre-engineering features to underpin a classification model. Active learning, on the other hand, is an approach that has been adopted to illustrate scenarios where a shortage of labelled data is available. They hypothesis that a learning algorithm can discriminate among relevant data to learn from, and hence perform better than traditional methods when lead data is available for training. The adoptions of deep artificial neural networks is another approach which is actively utilised by the research community [19]; authors point out that some of the benefits of implementing them are given due to the automatic identification of features of a given set of data. Similarly, several architectures are flexible to be adapted to new problems and data types [20]; a characteristic that make them convenient to scale. Nevertheless, some of the drawbacks of deep learning 
24 - Netzahualcoyotl Hernandez, Jens Lundström, Jesus Favela, lan McChesney, and Bert Arnrich are that they require a substantial amount of data to build a model, which can impact the computational cost.

Inferring human behaviour is challenging due to the rich variability of human action when performing a task. For instance, when a person experiences being observed, they will behave differently and therefore execute an activity in a different fashion compared to not being observed, (the Hawthorne effect [5]). In this regard, datasets captured in naturalistic conditions rather than controlled (i.e., in a laboratory) are challenging to obtain and more expensive in time and human effort. In this review, we grouped studies under four categories, aiming to characterise the likelihood that their experiments capture human behaviour more realistically. As observed in Section 5.4, the most popular approach is controlled condition studies, which consist of constraints that may obscure the true nature of the behaviour being studied. Given these results, we hypothesise that given the relative ease of using controlled condition data, new methodologies often adopt them to produce preliminary results. Whereas experiments conducted utilising naturalistic conditions, which can be expensive, represent evidence of a robust HAR methodology.

In this literature review, unsupervised HAR approaches refer to studies that do not require labelled data after the source domain has been defined. In this regard, one can argue that by introducing methods to recognise performed activities autonomously this alleviates, to some degree, the need for labelling or manual effort that is often required to transfer feature-representation or relational knowledge. By analysing the sub-set of unsupervised studies $(n=149)$, it can be found that the capacity to be autonomous extends to specific criteria which impact the model's HAR accuracy. For example, in this review, we found that $3 \%$ (i.e., 5) of the studies reported as unsupervised suggest an improvement on the model's accuracy upon variation on the task's distribution, $78 \%$ (i.e., 117) of the studies upon the presence of new participant profiles, $15 \%$ (i.e., 22) upon sensor setting variations, and 4\% (i.e., 6 ) 
Literature review on transfer learning for human activity recognition using mobile and wearable devices with environmental technology $• 25$

when new technology is introduced. Overall, the studies propose techniques that train HAR models and adapt to new circumstances thereafter (e.g., [28], [2]). Some studies utilise transfer learning strategies such as Teacher/Learner to automate an adaptation procedure to address the unsupervised approach. In [21], for example, they developed a system for HAR consisting of multiple views (e.g. one view from environmental sensors and another view from mobile sensors) collaborating to recognise unseen activities on an instance transfer level, reducing the dependency of labelled data.

Fully autonomous approaches aim at enabling classification models to detect key elements that reflect relevant activity patterns. In this study, only $4 \%$ (i.e., 6) of the unsupervised reviews were considered fully autonomous. For instance, Sulaimon et al., [4] investigated how to detect relevant changes to be considered in a HAR model, given that a variation on the participant's performance is followed by changes in the distribution of the input data attributes. Therefore, results suggest that such techniques drive the opportunity for building classification models to utilise naturalistic data. One of the drawbacks of some completely unsupervised autonomous approaches, however, is that they might require relevant manual knowledge engineering effort to define a comprehensive model. For instance, Riboni et al., designed an ontology built upon a factor of 29 times the number of activities to be recognised, i.e., 235 modelled classes to predict 8 human activities [46]. Whereas, in a supervised learning approach, a model might implement the same number of classes as those activities to be predicted.

About the conditions of how the data was collected for this subset of studies (i.e., unsupervised learning): $16 \%$ (i.e., 4) of the studies utilised a dataset which has been collected under naturalistic condition, $48 \%$ (i.e., 72 ) of the studies utilised controlled condition data, and $36 \%$ (i.e., 53) utilised naturalistic condition with controlled activities datasets. 
26 - Netzahualcoyotl Hernandez, Jens Lundström, Jesus Favela, Ian McChesney, and Bert Arnrich

\section{CONCLUSION}

In this paper, a literature review using the Okali and Schabram methodology is presented. Their approach intended to assure rigorous studies in the field of information systems. We systematically selected 170 papers published from 2014 to 2019. Selection criteria focused on solutions and techniques aligned to transfer learning and MWE computing technology. Our results describe the extent to which TL has been applied to HAR problems using MWE data, the amount of research conducted on transfer learning utilising mobile devices, and wearable electronic devices with environmental technologies. We also, investigated the three essential questions established by Saeedi et al. [48] regarding the development of transfer learning solutions; moreover, we introduce a fourth question (i.e., Where to transfer?) to explore the dimensions in which knowledge can be transferred (e.g., user, technology, locations). We conclude by presenting the results on the maturity of transfer learning based on the characteristics of the experiment's validation.

In summary, it can be concluded that there is a a growth in research in transfer learning techniques applied to HAR using data gathered from MWE, and that conducting experiments in naturalistic conditions utilising unsupervised HAR approaches with minimal manual knowledge engineering brings opportunities to the research community and for practical, real-world implementations.

\section{ABBREVIATIONS}

ADL Activity of daily living

AE Across environment

AL Across location

AR Across sampling rate

ART Adaptive resonance theory 
Literature review on transfer learning for human activity recognition using mobile and wearable devices with environmental technology $• 27$

AS Across sensor

AT Across task

AU Across user

CNN Deep Convolutional Networks

DNN Deep Neuronal Network

HAR Human activity recognition

IL Inductive learning

IoT Internet of Things

IS Information systems

MWE Mobile / Wearable / Environmental

T/L Teacher/Learner

TL Transductive learning

UL Unsupervised learning

\section{ACKNOWLEDGMENTS}

This project has received partial funding from the European Union's Horizon 2020 research and innovation programme under the Marie Skłodowska-Curie grant agreement No 734355.

\section{CONFLICT OF INTERESTS}

On behalf of all authors, the corresponding author states that there is no conflict of interest.

\section{REFERENCES}

[1] J. K. Aggarwal and Lu Xia. 2014. Human activity recognition from 3D data: A review. https://doi.org/10.1016/j.patrec. 2014.04.011 
[2] Mohammad Arif Ul Alam and Nirmalya Roy. 2017. Unseen Activity Recognitions: A Hierarchical Active Transfer Learning Approach. In Proceedings - International Conference on Distributed Computing Systems. https://doi.org/10.1109/ ICDCS.2017.264

[3] Geoff Appelboom, Elvis Camacho, Mickey E. Abraham, Samuel S. Bruce, Emmanuel L.P. Dumont, Brad E. Zacharia, Randy D’Amico, Justin Slomian, Jean Yves Reginster, Olivier Bruyère, and E. Sander Connolly. 2014. Smart wearable body sensors for patient self-assessment and monitoring. https://doi.org/10.1186/2049-3258-72-28

[4] Sulaimon A. Bashir, Andrei Petrovski, and Daniel Doolan. 2016. UDetect: Unsupervised Concept Change Detection for Mobile Activity Recognition. In Proceedings of the 14th International Conference on Advances in Mobile Computing and Multi Media (MoMM '16). ACM, New York, NY, USA, 20-27. https://doi.org/10.1145/3007120.3007144

[5] Fabrizio Benedetti, Elisa Carlino, and Alessandro Piedimonte. 2016. Increasing uncertainty in CNS clinical trials: the role of placebo, nocebo, and Hawthorne effects. https://doi.org/10.1016/S1474-4422(16)00066-1

[6] L. Benini, E. Farella, and C. Guiducci. 2006. Wireless sensor networks: Enabling technology for ambient intelligence. Microelectronics fournal 37, 12 (2006), 1639 - 1649. https://doi.org/10.1016/j.mejo.2006.04.021

[7] Eugen Berlin and Kristof Van Laerhoven. 2012. Detecting leisure activities with dense motif discovery. https://doi.org/ $10.1145 / 2370216.2370257$

[8] Gerald Bieber, Marian Haescher, and Matthias Vahl. 2013. Sensor requirements for activity recognition on smart watches. https://doi.org/10.1145/2504335.2504407

[9] S. N. Boualia and N. Essoukri Ben Amara. 2019. Pose-based Human Activity Recognition: a review. In 2019 15th International Wireless Communications Mobile Computing Conference (IWCMC). 1468-1475. https://doi.org/10.1109/ IWCMC.2019.8766694

[10] Jack P. Campbell, Vada A. Maxey, and William A. Watson. 1995. Hawthorne Effect: Implications for Prehospital Research. Annals of Emergency Medicine (1995). https://doi.org/10.1016/S0196-0644(95)70009-9

[11] Liangliang Cao, Zicheng Liu, and Thomas S. Huang. 2010. Cross-dataset action detection. In Proceedings of the IEEE Computer Society Conference on Computer Vision and Pattern Recognition. https://doi.org/10.1109/CVPR.2010.5539875

[12] A. Capponi, C. Fiandrino, B. Kantarci, L. Foschini, D. Kliazovich, and P. Bouvry. 2019. A Survey on Mobile Crowdsensing Systems: Challenges, Solutions, and Opportunities. IEEE Communications Surveys Tutorials 21, 3 (thirdquarter 2019), 2419-2465. https://doi.org/10.1109/COMST.2019.2914030 


\section{Literature review on transfer learning for human activity recognition using mobile and wearable devices with environmental technology $\quad 29$}

[13] Gail A. Carpenter, Stephen Grossberg, and John H. Reynolds. 1991. ARTMAP: Supervised real-time learning and classification of nonstationary data by a self-organizing neural network. Neural Networks (1991). https://doi.org/10. 1016/0893-6080(91)90012-T

[14] Liming Chen, Chris D. Nugent, and Hui Wang. 2012. A knowledge-driven approach to activity recognition in smart homes. IEEE Transactions on Knowledge and Data Engineering (2012). https://doi.org/10.1109/TKDE.2011.51

[15] Belkacem Chikhaoui, Shengrui Wang, and HéLèNe Pigot. 2012. ADR-SPLDA: Activity Discovery and Recognition by Combining Sequential Patterns and Latent Dirichlet Allocation. Pervasive Mob. Comput. 8, 6 (Dec. 2012), 845-862. https://doi.org/10.1016/j.pmcj.2012.08.004

[16] Diane Cook, Kyle D. Feuz, and Narayanan C. Krishnan. 2013. Transfer learning for activity recognition: a survey. Knowledge and Information Systems 36, 3 (01 Sep 2013), 537-556. https://doi.org/10.1007/s10115-013-0665-3

[17] J. K. Dhillon, Chandni, and A. K. S. Kushwaha. 2017. A recent survey for human activity recoginition based on deep learning approach. In 2017 Fourth International Conference on Image Information Processing (ICIIP). 1-6. https: //doi.org/10.1109/ICIIP.2017.8313715

[18] A. Dimitrievski, E. Zdravevski, P. Lameski, and V. Trajkovik. 2016. A survey of Ambient Assisted Living systems: Challenges and opportunities. In 2016 IEEE 12th International Conference on Intelligent Computer Communication and Processing (ICCP). 49-53. https://doi.org/10.1109/ICCP.2016.7737121

[19] Renjie Ding, Xue Li, Lanshun Nie, Jiazhen Li, Xiandong Si, Dianhui Chu, Guozhong Liu, and Dechen Zhan. 2019. Empirical Study and Improvement on Deep Transfer Learning for Human Activity Recognition. Sensors 19, 1 (2019). https://doi.org/10.3390/s19010057

[20] Xin Du, Katayoun Farrahi, and Mahesan Niranjan. 2019. Transfer Learning across Human Activities Using a Cascade Neural Network Architecture. In Proceedings of the 23rd International Symposium on Wearable Computers (ISWC '19). Association for Computing Machinery, New York, NY, USA, 35-44. https://doi.org/10.1145/3341163.3347730

[21] Kyle D. Feuz and Diane J. Cook. 2017. Collegial activity learning between heterogeneous sensors. Knowledge and Information Systems (2017). https://doi.org/10.1007/s10115-017-1043-3

[22] Dany Fortin-Simard, Jean Sebastien Bilodeau, Kevin Bouchard, Sebastien Gaboury, Bruno Bouchard, and Abdenour Bouzouane. 2015. Exploiting Passive RFID Technology for Activity Recognition in Smart Homes. IEEE Intelligent Systems (2015). https://doi.org/10.1109/MIS.2015.18 
30 • Netzahualcoyotl Hernandez, Jens Lundström, Jesus Favela, Ian McChesney, and Bert Arnrich

[23] Louise Francis. 2014. Unsupervised learning. In Predictive Modeling Applications in Actuarial Science: Volume I: Predictive Modeling Techniques. https://doi.org/10.1017/CBO9781139342674.012

[24] João Gama, Indrė Žliobaitè, Albert Bifet, Mykola Pechenizkiy, and Abdelhamid Bouchachia. 2014. A survey on concept drift adaptation. Comput. Surveys (2014). https://doi.org/10.1145/2523813

[25] Avinav Gupta and J. Priyadarshini. 2019. Smart Monitoring of Automatic Teller Machine-A Survey. In Proceedings of the Third International Conference on Microelectronics, Computing and Communication Systems, Vijay Nath and Jyotsna Kumar Mandal (Eds.). Springer Singapore, Singapore, 109-115.

[26] Hirotaka Hachiya, Masashi Sugiyama, and Naonori Ueda. 2012. Importance-weighted least-squares probabilistic classifier for covariate shift adaptation with application to human activity recognition. Neurocomputing (2012). https: //doi.org/10.1016/j.neucom.2011.09.016

[27] Derek Hao Hu and Qiang Yang. 2011. Transfer learning for activity recognition via sensor mapping. In IfCAI International foint Conference on Artificial Intelligence. https://doi.org/10.5591/978-1-57735-516-8/IJCAI11-328

[28] Lisha Hu, Yiqiang Chen, Shuangquan Wang, Jindong Wang, Jianfei Shen, Xinlong Jiang, and Zhiqi Shen. 2017. Less Annotation on Personalized Activity Recognition Using Context Data. In Proceedings - 13th IEEE International Conference on Ubiquitous Intelligence and Computing, 13th IEEE International Conference on Advanced and Trusted Computing, 16th IEEE International Conference on Scalable Computing and Communications, IEEE International Conference on Cloud and Big Data Computing, IEEE International Conference on Internet of People and IEEE Smart World Congress and Workshops, UICATC-ScalCom-CBDCom-IoP-SmartWorld 2016. https://doi.org/10.1109/UIC-ATC-ScalCom-CBDCom-IoP-SmartWorld. 2016.0066

[29] Tâm Huynh, Mario Fritz, and Bernt Schiele. 2008. Discovery of activity patterns using topic models. https://doi.org/10. $1145 / 1409635.1409638$

[30] A. K. Jain, M. N. Murty, and P. J. Flynn. 1999. Data clustering: a review. Comput. Surveys (1999). https://doi.org/10.1145/ 331499.331504

[31] H. Jiang, C. Cai, X. Ma, Y. Yang, and J. Liu. 2018. Smart Home Based on WiFi Sensing: A Survey. IEEE Access 6 (2018), 13317-13325. https://doi.org/10.1109/ACCESS.2018.2812887

[32] Charmi Jobanputra, Jatna Bavishi, and Nishant Doshi. 2019. Human Activity Recognition: A Survey. Procedia Computer Science 155 (2019), 698 - 703. https://doi.org/10.1016/j.procs.2019.08.100 The 16th International Conference on Mobile Systems and Pervasive Computing (MobiSPC 2019),The 14th International Conference on Future Networks and 
Literature review on transfer learning for human activity recognition using mobile and wearable devices with environmental technology • 31

Communications (FNC-2019),The 9th International Conference on Sustainable Energy Information Technology.

[33] Leyla Kazemi and Cyrus Shahabi. 2012. GeoCrowd: Enabling Query Answering with Spatial Crowdsourcing. In Proc. 20th ACM Int. Conf. Adv. Geogr. Inf. Syst. (SIGSPATIAL). https://doi.org/10.1145/2424321.2424346

[34] Yongjin Kwon, Kyuchang Kang, and Changseok Bae. 2014. Unsupervised learning for human activity recognition using smartphone sensors. Expert Systems with Applications (2014). https://doi.org/10.1016/j.eswa.2014.04.037

[35] N. D. Lane, E. Miluzzo, H. Lu, D. Peebles, T. Choudhury, and A. T. Campbell. 2010. A survey of mobile phone sensing. IEEE Communications Magazine 48, 9 (Sep. 2010), 140-150. https://doi.org/10.1109/MCOM.2010.5560598

[36] Óscar D. Lara and Miguel A. Labrador. 2013. A survey on human activity recognition using wearable sensors. IEEE Communications Surveys and Tutorials (2013). https://doi.org/10.1109/SURV.2012.110112.00192

[37] Falk Leichsenring. 2004. Randomized controlled versus naturalistic studies: A new research agenda. https://doi.org/10. 1521/bumc.68.2.137.35952

[38] Weibo Liu, Zidong Wang, Xiaohui Liu, Nianyin Zeng, Yurong Liu, and Fuad E. Alsaadi. 2017. A survey of deep neural network architectures and their applications. Neurocomputing (2017). https://doi.org/10.1016/j.neucom.2016.12.038

[39] T. Maekawa, D. Nakai, K. Ohara, and Y. Namioka. 2016. Toward practical factory activity recognition: Unsupervised understanding of repetitive assembly work in a factory. UbiComp 2016 (2016). https://doi.org/10.1145/2971648.2971721

[40] Ryszard S. Michalski. 1983. A theory and methodology of inductive learning. Artificial Intelligence (1983). https: //doi.org/10.1016/0004-3702(83)90016-4

[41] Francisco Javier Ordóñez Morales and Daniel Roggen. 2016. Deep Convolutional Feature Transfer across Mobile Activity Recognition Domains, Sensor Modalities and Locations. In Proceedings of the 2016 ACM International Symposium on Wearable Computers (ISWC '16). Association for Computing Machinery, New York, NY, USA, 92-99. https://doi.org/10. $1145 / 2971763.2971764$

[42] Chitu Okoli and Kira Schabram. 2011. A Guide to Conducting a Systematic Literature Review of Information Systems Research. (2011). https://doi.org/10.2139/ssrn.1954824

[43] Sinno Jialin Pan and Qiang Yang. 2010. A survey on transfer learning. https://doi.org/10.1109/TKDE.2009.191

[44] Qiang Yang and Sinno Jialin Pan. 2010. A Survey on Transfer Learning. IEEE Transactions on Knowledge and Data Engineering 22, 10 (2010), 1345--1359. https://doi.org/10.1109/TKDE.2009.191 
32 • Netzahualcoyotl Hernandez, Jens Lundström, Jesus Favela, Ian McChesney, and Bert Arnrich

[45] Reza Rawassizadeh, Blaine A. Price, and Marian Petre. 2014. Wearables: Has the Age of Smartwatches Finally Arrived? Commun. ACM (2014). https://doi.org/10.1145/2629633

[46] Daniele Riboni, Timo Sztyler, Gabriele Civitarese, and Heiner Stuckenschmidt. 2016. Unsupervised recognition of interleaved activities of daily living through ontological and probabilistic reasoning. https://doi.org/10.1145/2971648. 2971691

[47] Marcus Rohrbach, Sandra Ebert, and Bernt Schiele. 2013. Transfer learning in a transductive setting. Advances in neural information ... (2013).

[48] Ramyar Saeedi, Hassan Ghasemzadeh, and Assefaw H. Gebremedhin. 2016. Transfer learning algorithms for autonomous reconfiguration of wearable systems. In Proceedings - 2016 IEEE International Conference on Big Data, Big Data 2016. https://doi.org/10.1109/BigData.2016.7840648

[49] Dairazalia Sánchez, Monica Tentori, and Jesús Favela. 2008. Activity recognition for the smart hospital. IEEE Intelligent Systems (2008). https://doi.org/10.1109/MIS.2008.18

[50] Julia Seiter, Wei Chen Chiu, Mario Fritz, Oliver Amft, and Gerhard Tröster. 2015. Joint segmentation and activity discovery using semantic and temporal priors. In 2015 IEEE International Conference on Pervasive Computing and Communications, PerCom 2015. https://doi.org/10.1109/PERCOM.2015.7146511

[51] Dag I.K. Sjøberg, Jo E. Hannay, Ove Hansen, Vigdis By Kampenes, Amela Karahasanović, Nils Kristian Liborg, and Anette C. Rekdal. 2005. A survey of controlled experiments in software engineering. IEEE Transactions on Software Engineering (2005). https://doi.org/10.1109/TSE.2005.97

[52] M. Stikic, D. Larlus, and B. Schiele. 2009. Multi-graph Based Semi-supervised Learning for Activity Recognition. In 2009 International Symposium on Wearable Computers. 85-92. https://doi.org/10.1109/ISWC.2009.24

[53] Maja Stikic, Kristof Van Laerhoven, and Bernt Schiele. 2008. Exploring semi-supervised and active learning for activity recognition. In Proceedings - International Symposium on Wearable Computers, ISWC. https://doi.org/10.1109/ISWC.2008. 4911590

[54] Fabio A. Storm, Ben W. Heller, and Claudia Mazzà. 2015. Step detection and activity recognition accuracy of seven physical activity monitors. PLoS ONE (2015). https://doi.org/10.1371/journal.pone.0118723

[55] Xing Su, Hanghang Tong, and Ping Ji. 2014. Activity recognition with smartphone sensors. Tsinghua Science and Technology (2014). https://doi.org/10.1109/TST.2014.6838194 


\section{Literature review on transfer learning for human activity recognition using mobile and wearable devices with environmental technology $• 33$}

[56] Myung Kyung Suh, Chien An Chen, Jonathan Woodbridge, Michael Kai Tu, Jung In Kim, Ani Nahapetian, Lorraine S. Evangelista, and Majid Sarrafzadeh. 2011. A remote patient monitoring system for congestive heart failure. In fournal of Medical Systems. https://doi.org/10.1007/s10916-011-9733-y

[57] Shiliang Sun. 2013. A survey of multi-view machine learning. https://doi.org/10.1007/s00521-013-1362-6

[58] Kristin Taraldsen, Sebastien F M Chastin, Ingrid I. Riphagen, Beatrix Vereijken, and Jorunn L. Helbostad. 2012. Physical activity monitoring by use of accelerometer-based body-worn sensors in older adults: A systematic literature review of current knowledge and applications. https://doi.org/10.1016/j.maturitas.2011.11.003

[59] Alireza Vahdatpour, Navid Amini, and Majid Sarrafzadeh. 2011. On-body device localization for health and medical monitoring applications. In 2011 IEEE International Conference on Pervasive Computing and Communications, PerCom 2011. https://doi.org/10.1109/PERCOM.2011.5767593

[60] T van Kasteren, G Englebienne, and BJA Kröse. 2008. Recognizing Activities in Multiple Contexts using Transfer Learning. AAAI AI in Eldercare Symposium (2008).

[61] Jiwei Wang, Yiqiang Chen, Yang Gu, Yunlong Xiao, and Haonan Pan. 2018. SensoryGANs: An Effective Generative Adversarial Framework for Sensor-based Human Activity Recognition. In Proceedings of the International foint Conference on Neural Networks. https://doi.org/10.1109/IJCNN.2018.8489106

[62] Che Chang Yang and Yeh Liang Hsu. 2010. A review of accelerometry-based wearable motion detectors for physical activity monitoring. https://doi.org/10.3390/s100807772

[63] Xu Cheng Yin, Chun Yang, Wei Yi Pei, and Hong Wei Hao. 2014. Shallow classification or deep learning: An experimental study. In Proceedings - International Conference on Pattern Recognition. https://doi.org/10.1109/ICPR.2014.333

[64] S. Zhu, J. Xu, H. Guo, Q. Liu, S. Wu, and H. Wang. 2018. Indoor Human Activity Recognition Based on Ambient Radar with Signal Processing and Machine Learning. In 2018 IEEE International Conference on Communications (ICC). 1-6. https://doi.org/10.1109/ICC.2018.8422107 